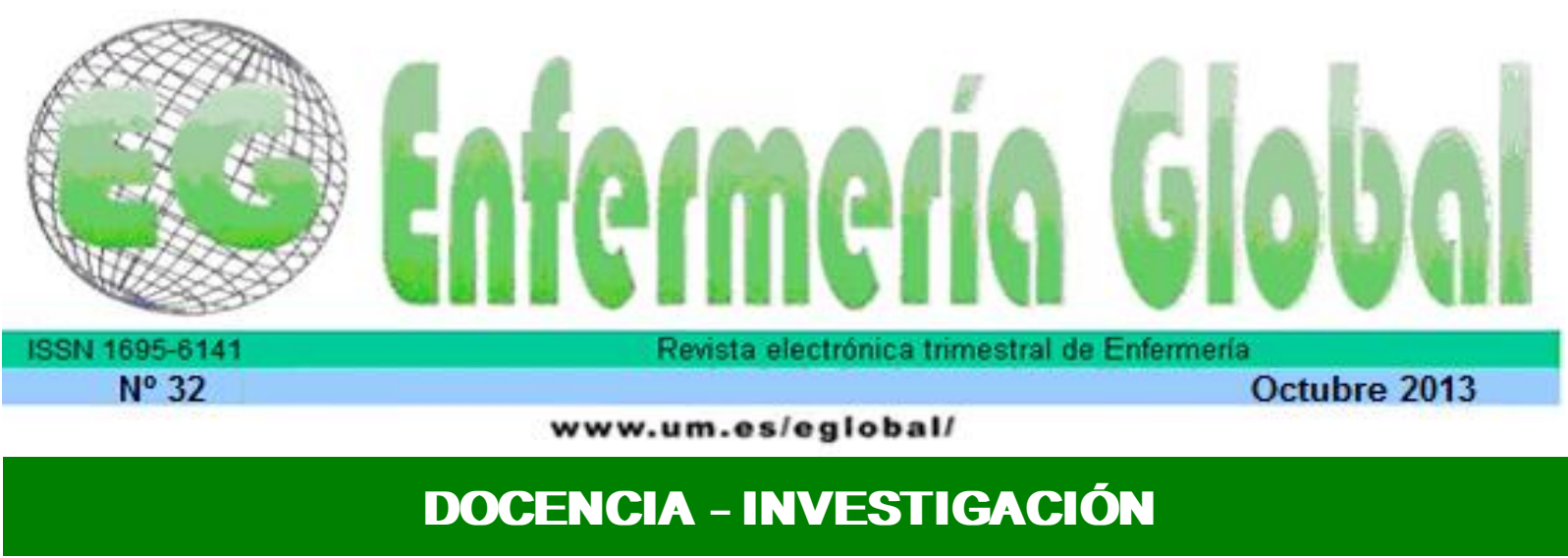

\title{
Estrés del paciente en UCl: visión de los pacientes y del equipo de enfermería
}

Estresse do paciente em UTI: visão de pacientes e equipe de enfermagem

ICU patient stress: vision patients and the nursing team

\section{${ }^{*}$ Fernandes da Cruz Silva, Leticia ${ }^{* *}$ Machado, Regimar Carla *** Giaretta, Vania Maria de Araújo ****Salazar Posso, Maria Belén.}

\begin{abstract}
*Enfermera del Hospital Municipal Dr. José de Carvalho Florence. Especialista en Enfermería en Cuidados Críticos/ Cardiología. **Doctora en Enfermería. Profesora del Curso de Pos-Graduación de Enfermería en Cuidados Críticos/ Cardiología. E-mail: regimarcarla@yahoo.com.br ${ }^{* * *}$ Enfermera. Máster en Ingeniería Biomédica. Profesora del Curso de Pos- Graduación de Enfermería en Cuidados Críticos/ Cardiología ${ }^{* * * *}$ Doctora en Enfermería. Profesora del Departamento de Enfermería. Universidad Vale do Paraíba- UNIVAP - São José dos Campos (SP), Brasil.
\end{abstract}

\author{
Palabras clave: Unidades de Cuidados Intensivos; Estrés; Enfermería. \\ Palavras chave: Unidade de terapia intensiva; Estresse; Enfermagem \\ Keywords: Intensive care units; Stres; Nursing.
}

\section{RESUMEN}

Objetivos: Identificar los factores estresantes en pacientes ingresados en dos Unidades de Cuidados Críticos, levantar los mecanismos utilizados por el equipo de enfermería para minimizar los factores que desencadenan el estrés al pacientes y comparar las contestaciones de los pacientes y del equipo de enfermería según las variables del grado de estrés.

Métodos: Estudio descriptivo, exploratorio y cuantitativo. La muestra estuvo compuesta de 16 pacientes y 50 colaboradores de enfermería. La recolección de datos mediante cuestionarios validados.

Resultados: Los factores más estresantes para los pacientes fueron relacionados con quedarse mirando para el techo; no tener privacidad; no saber en qué día está, y escuchar los gemidos de otros pacientes. Los colaboradores de enfermería relataron realizar importantes acciones de humanización en su trabajo. Cuando comparadas las variables del estrés del paciente por la visión del equipo de enfermería, hubo diferencia significativa en las variables cualitativas del estrés.

Conclusiones: El ingreso en la UCI fue considerado como no estresante a poco estresante para el paciente. El profesional de enfermería tiene la capacidad de proyectarse y sensibilizarse hasta el punto de saber en qué medida determinado factor es estresante para el paciente. Los profesionales hacen su 
trabajo de manera eficiente, cuidadosa y con respeto a las normas de humanización, sin embargo este hecho no siempre es eficaz para resolver los problemas levantados de todos los pacientes.

\section{RESUMO}

Objetivos: Identificar fatores que atuam como estressores em pacientes internados em duas Unidades de Cuidados Intensivos, levantar os mecanismos utilizados pela equipe de enfermagem para amenizar os fatores desencadeantes de estresse ao paciente e comparar as respostas dos pacientes e da equipe de enfermagem segundo as variáveis do grau do estresse.

Métodos: Estudo descritivo, exploratório e quantitativo. Amostra com 16 pacientes e 50 colaboradores de enfermagem. Coleta de dados através de questionários validados.

Resultados: Os fatores mais estressantes para os pacientes foram relacionados a ficar olhando para o teto, a não ter privacidade, não saber em qual dia está, e escutar os gemidos de outros pacientes. Os colaboradores de enfermagem relataram realizar importantes ações de humanização no seu trabalho. Quando comparadas as variáveis do estresse do paciente pela visão da equipe de enfermagem, houve diferença significativa nas variáveis qualitativas do estresse.

Conclusões: A internação em UTI foi considerada como não estressante a pouco estressante para o paciente. O profissional de enfermagem tem a capacidade de projetar-se e sensibilizar-se a ponto de saber quanto determinado fator é estressante para o paciente.Os profissionais estão fazendo seu trabalho de maneira eficiente, cuidadosa e com respeito às normas de humanização, mas este fato nem sempre é eficaz para sanar os problemas levantados de todos os pacientes.

\section{ABSTRACT}

Objectives: To identify factors that act as stressors in two patients in Critical Care Units, to increase the mechanisms used by the nursing staff to mitigate the factors that cause stress to the patient and compare the responses of patients and nursing staff according to the variables degree of stress.

Methods: A descriptive, exploratory and quantitative. Sample of 16 patients and 50 nursing staff. Collection of data through validated questionnaires.

Results: The most stressful factors related to patients were to be staring at the ceiling, not to have privacy, not to know which day is, and hear the groans of other patients. The nursing staff reported to achieve important humanization actions in their work. When comparing the variables of stress for the patient's view of nursing staff, there were significant differences in qualitative variables of stress.

Conclusions: The ICU admission was considered as non-stressful little stressful for the patient. The nursing professional has the ability to project themselves and raise awareness to the point of knowing how a given factor is stressful for paciente.Os professionals are doing their job efficiently, carefully and with respect for the norms of humanization, but this fact is not always effective to remedy the problems of all patients.

\section{INTRODUCCIÓN}

El ingreso hospitalario favorece el estrés del paciente y de su familia. Sin embargo, puede exacerbarse cuando la situación del paciente sugiere un ingreso en una Unidad de Terapia Intensiva (UTI). ${ }^{(1)}$

No obstante, el estrés es considerado un problema relevante de la sociedad moderna, aunque produce diversas alteraciones fisiológicas, como la taquicardia, el aumento del volumen sanguíneo, la presión arterial acentuada, el aumento del estímulo neural, el cambio en la frecuencia respiratoria, la retención de sodio; en fin, una redistribución de fuentes de energía a la espera de una agresión. Por lo tanto, en caso de que realmente exista un peligro inminente, esta modificación fisiológica es ventajosa y 
beneficiosa mediante la situación, pero si el estado de estrés se mantuviera durante un largo período de tiempo, podría causar daños irreversibles. ${ }^{(2,3)}$

Antes de tratar el tema, vale definir el término estresor, que es considerado como un estímulo o situación que produce respuesta al estrés. ${ }^{(4)}$ Este, a su vez, exige una adaptación física y/o psicológica en el individuo. ${ }^{(5)}$

Cabe destacar que para que haya estrés, se necesita la existencia de un factor estresante o estresor. Estos pueden presentar diversas características en la causa del estrés, pero el cuerpo se manifiesta de la misma manera, sea cual fuere la fuente. ${ }^{(3)}$

La identificación de los estresores en el paciente es de extrema importancia para promover la humanización del ambiente de la UTI, pues posibilita que el enfermero pueda actuar ante esos factores, aplicando las medidas necesarias. ${ }^{(6)}$

Los enfermeros y el equipo de enfermería están muy cerca del paciente, siendo necesario enfocar su atención en las necesidades bio-psico-socio-espirituales, con cuidados individualizados. ${ }^{(7-9)}$ No obstante, el cuidado individualizado organiza la calidad de la atención, con la finalidad de minimizar o eliminar los factores negativos, facilitando así el proceso de recuperación, disminuyendo el tiempo de ingreso, y por consiguiente, los índices de infección hospitalaria. ${ }^{(10)}$

Ante lo expuesto, se observa que la presencia de pacientes lúcidos y despiertos en la UTI puede traer señales de estrés social y psicológico, y hasta comprometer su evolución clínica, considerando que los factores de estrés pueden producirse para atenuarlos o prevenirlos. Por lo tanto, en este estudio se pretende identificar factores que actúan como estresores en pacientes internados en la UTI y establecer los mecanismos utilizados por el equipo de enfermería para suavizar dichos factores desencadenantes del estrés.

\section{MÉTODOS}

Estudio descriptivo, exploratorio, de procedimiento transversal, con enfoque en la incidencia y/o en la prevalencia de una o más variables, con abordajes cuantitativos.

Con el objetivo de proteger los sujetos del estudio, el protocolo de investigación fue enviado al Comité de Ética en el área de Investigación de la Universidad do Vale de Paraíba, y aprobado bajo el nº H196/CEP/2010.

En primer lugar, los sujetos de este estudio constaron de 16 pacientes internados en Unidades de Cuidados Intensivos de dos instituciones diferentes, pero con las mismas características en cuanto al perfil de las unidades. Todos los pacientes que aceptaron participar del estudio firmaron el Término de Consentimiento Libre y Claro (TCLE, por sus siglas en portugués).

Se han incluido aquellos pacientes ingresados con período superior a 48 horas, cuyas edades superen los 18 años y en condiciones clínicas que permitan la respuesta del instrumento utilizado para la recopilación de datos.

Se establecieron como criterios de exclusión, los pacientes que presentaban descenso en el nivel de conciencia y clínica general, por tener dificultades de comprensión del estudio. 
Para la caracterización sociodemográfica de los sujetos, el instrumento de recolección de datos fue adaptado de otros dos estudios ${ }^{(9,11)}$ y para evaluación de los estresores se utilizó la Escala de Estresores en Terapia Intensiva (EETI) en la versión traducida y certificada al portugués. ${ }^{(12)}$

Fueron seleccionadas 31 preguntas que abordaban diversos factores generadores de estrés en la unidad de terapia intensiva ofreciendo opciones libres de respuestas al participante. Por lo tanto, para la clasificación de los factores considerados estresantes, se estableció un puntaje utilizando una escala tipo Likert. La escala correspondía a 4 puntos, de los cuales (1) significaba no estresante, (2) poco estresante, (3) estresante y (4) muy estresante.

Para responder al segundo objetivo del estudio fueron incluidos 50 colaboradores del área de enfermería de las mismas unidades que prestaron cuidados a los mismos pacientes seleccionados. Los colaboradores participaron del estudio luego de haber firmado el TCLE. Fueron establecidos como criterios de exclusión los colaboradores que no se desempeñaban en la asistencia con los pacientes del estudio y los que no aceptaron participar de la investigación.

Se utilizó el instrumento de la EETI en su versión traducida y certificada al portugués (12), y adaptado para el relevo de los datos con los profesionales colaboradores de enfermería.

Fueron seleccionadas 10 preguntas que abordaban diversos factores generadores de estrés en el paciente. Dichos factores están relacionados directa o indirectamente a la asistencia de enfermería en la unidad de cuidados críticos, ofreciendo libres opciones de respuestas al participante. Las preguntas evaluaron la asistencia prestada y la visión del equipo de enfermería en relación al grado de interés causado al paciente en determinadas situaciones potencialmente evitables, en que son establecidas situaciones de su vida diaria laboral y la actitud tomada por medio de los hechos. Vale ratificar que para la clasificación de los factores, considerados como estresantes, también se ha establecido un puntaje utilizando una escala tipo Likert de 4 puntos.

Se realizaron entrevistas con pacientes y colaboradores del área de enfermería, pertenecientes a la muestra durante el período de marzo a junio de 2011. Las entrevistas fueron agendadas y realizadas individualmente, respetando la disponibilidad de los participantes.

Los datos fueron procesados con la ayuda de los programas Microsoft Exce ${ }^{\circledR}$ y Microsoft Word $^{\circledR}$ en las versiones Office 2003. Se analizaron bajo la orientación estadística del programa SAS system v8 por medio de la estadística descriptiva (media, mediana, desvío estándar, frecuencias y porcentuales).

Para verificar si había una diferencia estadísticamente significativa respecto de la sensación de Estrés entre los Grupos de Pacientes y de Enfermeros, se optó por las pruebas estadísticas Chi-Cuadrado y Exacto de Fisher. Sin embargo, para verificar si había una diferencia estadísticamente significativa entre el Grupo de Pacientes y el Grupo de Enfermeros respecto del Grado de Estrés de cada factor analizado, se utilizó la Prueba de la Suma de Puestos de Wilcoxon, también conocido como Prueba de Mann-Whitney para comparación entre dos muestras independientes. 


\section{RESULTADOS}

\section{Variables referidas a los pacientes}

La edad promedio de los sujetos de la investigación fue de 50 años, con una variación entre 18 y 78 años, la mayoría pertenecientes al género masculino y el índice de escolaridad fue entre 9 y 12 años de estudios (descritas en la Tabla 1).

Tabla 1 - Caracterización sociodemográfica de los pacientes internados en las unidades de cuidados intensivos. São José dos Campos, 2011.

\begin{tabular}{lccc}
\hline Variable & IntervaloPromedio & $\mathbf{n}$ & $\%$ \\
\hline Edad (años) & $18---7850$ & 16 & 100,0 \\
& & & \\
Género & & 5 & 31,3 \\
Femenino & & 11 & 68,7 \\
Masculino & & $\mathbf{1 6}$ & $\mathbf{1 0 0 , 0}$ \\
Total & & & \\
\hline
\end{tabular}

\section{Estado civil}

Casado (a)

Soltero (a)

Viudo (a)

Separado/Divorciado (a)

Total

$\begin{array}{rr}4 & 25,0 \\ 3 & 18,8 \\ 6 & 37,5 \\ 3 & 18,7 \\ \mathbf{1 6} & \mathbf{1 0 0 , 0}\end{array}$

\section{Escolaridad}

Hasta 4 años

De 5 a 8 años

De 9 a 12 años

A partir de los 12 años

Total

$\begin{array}{rr}4 & 25,0 \\ 2 & 12,5 \\ 9 & 56,2 \\ 1 & 6,3 \\ \mathbf{1 6} & \mathbf{1 0 0 , 0}\end{array}$

\section{Situación profesional}

Activo

Jubilado

Ama de casa

Desempleado

Separado por Problemas de Salud

Total

$\begin{array}{rr}7 & 43,6 \\ 6 & 37,5 \\ 1 & 6,3 \\ 1 & 6,3 \\ 1 & 6,3 \\ \mathbf{1 6} & \mathbf{1 0 0 , 0}\end{array}$

El instrumento de la EETI fue seleccionado para la evaluación de los estresores en los pacientes internados. El Alfa de Cronbach, medida de la confiabilidad del instrumento fue de 0,90 , reflejando así la alta consistencia interna de los ítems presentes en la escala. 
El intervalo posible para la escala era de 31 (31 preguntas $\times 1$ ) hasta $124(31 \times 4)$, siendo que, cuanto mayor es la sumatoria de los ítems, mayor era el estrés notado en la UTI. En los individuos voluntarios se ha observado un valor promedio de 54,2 con un intervalo de 34 a 83.

Fueron evaluados los resultados de esa escala en cuanto al promedio de las respuestas a los ítems, es decir, la suma de los dos puntos establecidos en todos los ítems divididos por 31 (total de ítems de la escala). Por lo tanto, en el estudio se obtuvo el valor de 1,75, con intervalo de los promedios entre 1,10 y 2,68 . Al considerar los valores de estrés en la escala de Likert, los pacientes entrevistados consideraron el ingreso como una experiencia entre no estresante y poco estresante.

La posición de las puntuaciones dadas a los ítems de la escala de los pacientes permitió establecer cuáles son los ítems considerados como mayores fuentes de estrés, presentados en la Tabla 2. 
Tabla 2 - Relatos de los pacientes respecto de los factores estresantes en la unidad de cuidados intensivos. São José dos Campos, 2011.

\begin{tabular}{lcrr}
\hline Estresores & Posición & Promedio & D.P. \\
\hline Mirar al techo & 1 & 2,44 & 1,26 \\
No tener privacidad & 2 & 2,31 & 1,35 \\
No saber que día es hoy & 3 & 2,13 & 1,02 \\
Escuchar el gemido de otros pacientes & 3 & 2,13 & 1,36 \\
Sentir que no se tiene control de sí mismo & 4 & 2,06 & 1,29 \\
No saber cuando las cosas serán hechas & 4 & 2,06 & 1,24 \\
Sonidos y ruidos desconocidos & 4 & 2,06 & 1,24 \\
No saber la hora & 5 & 2,00 & 1,26 \\
Sentir dolor & 6 & 1,94 & 1,24 \\
Escuchar el ruido y alarma de los equipamientos & 6 & 1,94 & 1,29 \\
No explicar sobre el tratamiento & 7 & 1,88 & 1,26 \\
Escuchar la alarma del monitor cardíaco & 7 & 1,88 & 1,15 \\
Percibir olores extraños & 8 & 1,81 & 1,22 \\
Escuchar al equipo hablando con términos & & & \\
desconocidos & 8 & 1,81 & 1,17 \\
No lograr mover manos y brazos debido a vías & & & \\
intravenosas & 9 & 1,69 & 1,25 \\
Hombres y mujeres internados en el mismo & & & \\
ambiente & 9 & 1,69 & 1,20 \\
Enfermeros y médicos hablando alto & 9 & 1,69 & 1,14 \\
Tener sed & 10 & 1,63 & 1,20 \\
Usar oxígeno & 10 & 1,63 & 0,96 \\
Ver las bolsas de suero colgadas sobre la cabeza & 10 & 1,63 & 1,20 \\
Estar en un ambiente muy caliente o muy frío & 10 & 1,63 & 0,96 \\
No lograr dormir durante las noches & 11 & 1,56 & 1,09 \\
Ser inyectado & 11 & 1,56 & 0,96 \\
Máquinas extrañas alrededor & 11 & 1,56 & 0,96 \\
Estar bajo el cuidado de médicos desconocidos & 11 & 1,56 & 1,21 \\
Medir la presión arterial muchas veces al día & 12 & 1,50 & 0,97 \\
Ser despertado por el equipo de enfermería & 13 & 1,44 & 0,81 \\
Cama y/o almohada no son confortables & 14 & 1,31 & 0,87 \\
Ver cuidados médicos y de enfermería realizados & & 1,31 & 0,87 \\
en otros pacientes & 14 & 1,13 & 0,31 \\
El enfermero no se presenta por su nombre & & & 0,70 \\
Escuchar que suena el teléfono & & & \\
\hline
\end{tabular}

\section{Variables Referidas a los Colaboradores de Enfermería}

La edad promedio de los participantes fue de 32 años, con una variación de entre 22 y 47 años. La mayoría pertenecían al género femenino. La formación profesional fue bien distribuida entre las tres categorías de enfermería, siendo la mayor parte técnicos de enfermería. El tiempo de formación fue elevado, estando comprendido, en gran parte, entre 6 y 10 años. 
Fueron seleccionadas 10 preguntas que abordaban los mecanismos utilizados por el equipo de enfermería para amenizar los factores desencadenantes de estrés a los pacientes internados en las Unidades de Cuidados Intensivos.

Tabla 3 - Resultados de las acciones de humanización realizadas por los colaboradores de enfermería frente al paciente internado en la Unidad de Cuidados Intensivos. São José dos Campos, 2011.

\begin{tabular}{|c|c|c|c|}
\hline Acciones & Sí \% (n) & No \% (n) A & unas veces \\
\hline $\begin{array}{l}\text { Presentarse con el nombre } \\
\text { en el primer contacto con el paciente }\end{array}$ & $76 \%(38)$ & $2 \%(1)$ & $22 \%(11)$ \\
\hline $\begin{array}{l}\text { Explicar al paciente respecto del } \\
\text { trato/procedimiento que se realizará }\end{array}$ & $86 \%(43)$ & $0 \%(0)$ & $14 \%(7)$ \\
\hline $\begin{array}{l}\text { Evitar informaciones técnicas que puedan } \\
\text { dificultar el entendimiento del trato con } \\
\text { el paciente }\end{array}$ & $78 \%(39)$ & $8 \%(4)$ & $14 \%(7)$ \\
\hline $\begin{array}{l}\text { Preocuparse en avisar con anticipación } \\
\text { (cuando sea posible) respecto de cualquier } \\
\text { procedimiento/intervención que se realizará } \\
\text { con el paciente, y explicar su finalidad }\end{array}$ & $88 \%(44)$ & $2 \%(1)$ & $10 \%(5)$ \\
\hline $\begin{array}{l}\text { Estar atento de no hablar muy alto en } \\
\text { la sala de cuidados críticos }\end{array}$ & $80 \%(40)$ & $8 \%(4)$ & $12 \%(6)$ \\
\hline $\begin{array}{l}\text { Preocuparse por la privacidad al realizar } \\
\text { una intervención }\end{array}$ & $86 \%(43)$ & $0 \%(0)$ & $14 \%(7)$ \\
\hline $\begin{array}{l}\text { Cuidar que el paciente no } \\
\text { vea los cuidados médicos y } \\
\text { de enfermería realizados con } \\
\text { otros pacientes }\end{array}$ & $76 \%(38)$ & $0 \%(0)$ & $24 \%(12)$ \\
\hline $\begin{array}{l}\text { Orientar al paciente despierto } \\
\text { sobre el día de la semana y el horario }\end{array}$ & $52 \%(26)$ & $10 \%(5)$ & $38 \%(19)$ \\
\hline Preocuparse por la sed del paciente & $72 \%(36)$ & $4 \%(2)$ & $24 \%(12)$ \\
\hline $\begin{array}{l}\text { Poseer algún método de evaluación del } \\
\text { dolor de la institución que desempeña }\end{array}$ & $60 \%(30)$ & $38 \%(19)^{*}$ & \\
\hline
\end{tabular}

Incluso se realizó la comparación de las respuestas de los pacientes con las respuestas del equipo de enfermería, de acuerdo con las variables cualitativas y cuantitativas del grado de estrés, y estas se presentan en los Cuadros 1 y 2. 
Cuadro 1 - Comparación de las respuestas de los pacientes con las respuestas del equipo de enfermería, de acuerdo con las variables cualitativas del estrés. São José dos Campos, 2011.

\begin{tabular}{|c|c|c|c|c|}
\hline \multicolumn{2}{|c|}{ Variables cualitativas } & \multicolumn{3}{|c|}{ Prueba Exacta de Fisher/ Chi-Cuadrado } \\
\hline $\begin{array}{l}\text { Respuesta del } \\
\text { paciente }\end{array}$ & $\begin{array}{l}\text { Desempeño } \\
\text { del equipo de } \\
\text { enfermería }\end{array}$ & $\begin{array}{c}\text { Nivel de } \\
\text { significancia }\end{array}$ & $\mathbf{P}$ & Conclusión \\
\hline $\begin{array}{l}\text { El enfermero no } \\
\text { se presenta por } \\
\text { su nombre }\end{array}$ & $\begin{array}{l}\text { En todo primer } \\
\text { contacto con el } \\
\text { paciente, } \\
\text { presentarse por } \\
\text { su nombre }\end{array}$ & 0,05 & $p=0,0189$ & $\begin{array}{c}\text { Existe } \\
\text { diferencia } \\
\text { significativa } \\
\text { en la } \\
\text { proporción de } \\
\text { respuestas }\end{array}$ \\
\hline $\begin{array}{l}\text { No explicar } \\
\text { sobre el } \\
\text { tratamiento }\end{array}$ & $\begin{array}{l}\text { Explicarle } \\
\text { siempre al } \\
\text { paciente sobre } \\
\text { el tratamiento/ } \\
\text { procedimiento } \\
\text { que se } \\
\text { realizará }\end{array}$ & 0,05 & $p=0,0686$ & $\begin{array}{l}\text { No existe } \\
\text { diferencia } \\
\text { significativa }\end{array}$ \\
\hline $\begin{array}{l}\text { Hablarle al } \\
\text { paciente con } \\
\text { términos } \\
\text { desconocidos }\end{array}$ & $\begin{array}{l}\text { Preocuparse } \\
\text { por no hablarle } \\
\text { al paciente con } \\
\text { términos } \\
\text { técnicos }\end{array}$ & 0,05 & $p=0,7104$ & $\begin{array}{l}\text { No existe } \\
\text { diferencia } \\
\text { significativa }\end{array}$ \\
\hline $\begin{array}{l}\text { No saber } \\
\text { cuándo se } \\
\text { realizarán las } \\
\text { cosas }\end{array}$ & $\begin{array}{l}\text { Preocuparse } \\
\text { en comunicar } \\
\text { con } \\
\text { anticipación } \\
\text { (cuando sea } \\
\text { posible) sobre } \\
\text { cualquier } \\
\text { procedimiento/ } \\
\text { intervención } \\
\text { que se } \\
\text { realizará con el } \\
\text { paciente, y } \\
\text { explicar su } \\
\text { finalidad. } \\
\end{array}$ & 0,05 & $p=0,0511$ & $\begin{array}{l}\text { No existe } \\
\text { diferencia } \\
\text { significativa }\end{array}$ \\
\hline $\begin{array}{l}\text { Enfermeros y } \\
\text { médicos } \\
\text { hablando muy } \\
\text { alto }\end{array}$ & $\begin{array}{l}\text { Estar atento de } \\
\text { no hablar muy } \\
\text { alto en la sala } \\
\text { de cuidados } \\
\text { intensivos }\end{array}$ & 0,05 & $p=0,0053$ & $\begin{array}{c}\text { Existe } \\
\text { diferencia } \\
\text { significativa } \\
\text { en la } \\
\text { proporción de } \\
\text { respuestas } \\
\end{array}$ \\
\hline $\begin{array}{l}\text { No tener } \\
\text { privacidad }\end{array}$ & $\begin{array}{l}\text { Preocuparse } \\
\text { por la } \\
\text { privacidad al } \\
\text { realizar una } \\
\text { intervención }\end{array}$ & 0,05 & $p=0,1893$ & $\begin{array}{c}\text { No existe } \\
\text { diferencia } \\
\text { significativa }\end{array}$ \\
\hline
\end{tabular}




\begin{tabular}{|l|l|l|l|c|}
\hline $\begin{array}{l}\text { Observar los } \\
\text { cuidados } \\
\text { médicos y de } \\
\text { enfermería } \\
\text { realizados con } \\
\text { otros pacientes }\end{array}$ & $\begin{array}{l}\text { Estar siempre } \\
\text { atento de que } \\
\text { un paciente no } \\
\text { observe los } \\
\text { cuidados } \\
\text { médicos y de } \\
\text { enfermería } \\
\text { realizados con } \\
\text { otros pacientes }\end{array}$ & 0,05 & $\mathrm{p}<0,0001$ & $\begin{array}{c}\text { Existe } \\
\text { diferencia } \\
\text { significativa } \\
\text { en la } \\
\text { proporción de } \\
\text { respuestas. }\end{array}$ \\
\hline $\begin{array}{l}\text { No saber qué } \\
\text { día es hoy }\end{array}$ & $\begin{array}{l}\text { Orientar al } \\
\text { paciente } \\
\text { despierto } \\
\text { respecto del } \\
\text { día de la } \\
\text { semana y el } \\
\text { horario. }\end{array}$ & 0,05 & $\mathrm{p}=0,5237$ & $\begin{array}{c}\text { No existe } \\
\text { diferencia } \\
\text { significativa }\end{array}$ \\
\hline Tener sed & $\begin{array}{l}\text { Preocuparse } \\
\text { por la sed y la } \\
\text { hidratación del } \\
\text { paciente }\end{array}$ & 0,05 & $\mathrm{p}=0,0486$ & $\begin{array}{c}\text { Existe } \\
\text { diferencia } \\
\text { significativa } \\
\text { en la } \\
\text { proporción de } \\
\text { respuestas. }\end{array}$ \\
\hline Sentir dolor & $\begin{array}{l}\text { Poseer algún } \\
\text { método de } \\
\text { evaluación del } \\
\text { dolor en la } \\
\text { institución que } \\
\text { se desempeña }\end{array}$ & 0,05 & $\mathrm{p}=0,0642$ & $\begin{array}{c}\text { No existe } \\
\text { diferencia } \\
\text { significativa }\end{array}$ \\
\hline
\end{tabular}

Cuadro 2 - Comparación de las respuestas de los pacientes con las respuestas del equipo de enfermería, de acuerdo con las variables cuantitativas del estrés. São José dos Campos, 2011.

\begin{tabular}{|c|c|c|c|c|}
\hline \multicolumn{2}{|c|}{ Variables cuantitativas } & \multicolumn{3}{|c|}{ Prueba de Mann-Whitney } \\
\hline $\begin{array}{l}\text { Respuesta } \\
\text { del paciente }\end{array}$ & $\begin{array}{l}\text { Desempeño del equipo } \\
\text { de enfermería }\end{array}$ & $\begin{array}{c}\text { Nivel de } \\
\text { significancia }\end{array}$ & $\mathbf{P}$ & Conclusión \\
\hline $\begin{array}{l}\text { El enfermero } \\
\text { no se } \\
\text { presenta por } \\
\text { su nombre }\end{array}$ & $\begin{array}{l}\text { En todo primer contacto } \\
\text { con el paciente, } \\
\text { presentarse por su } \\
\text { nombre }\end{array}$ & 0,05 & $\begin{array}{c}\mathrm{p} \\
=0,7237\end{array}$ & $\begin{array}{l}\text { No existe } \\
\text { diferencia } \\
\text { significativa }\end{array}$ \\
\hline $\begin{array}{l}\text { No dar } \\
\text { explicaciones } \\
\text { sobre el } \\
\text { tratamiento }\end{array}$ & $\begin{array}{l}\text { Explicarle siempre al } \\
\text { paciente respecto del } \\
\text { tratamiento/procedimiento } \\
\text { que se realizará. }\end{array}$ & 0,05 & $\begin{array}{c}\mathrm{p} \\
=0,5505\end{array}$ & $\begin{array}{l}\text { No existe } \\
\text { diferencia } \\
\text { significativa }\end{array}$ \\
\hline $\begin{array}{l}\text { Hablarle al } \\
\text { paciente con } \\
\text { términos } \\
\text { desconocidos }\end{array}$ & $\begin{array}{l}\text { Preocuparse por no } \\
\text { hablarle al paciente con } \\
\text { términos técnicos. }\end{array}$ & 0,05 & $\begin{array}{c}\mathrm{p} \\
=0,7576\end{array}$ & $\begin{array}{l}\text { No existe } \\
\text { diferencia } \\
\text { significativa }\end{array}$ \\
\hline
\end{tabular}




\begin{tabular}{|c|c|c|c|c|}
\hline $\begin{array}{l}\text { No saber } \\
\text { cuándo se } \\
\text { harán las } \\
\text { cosas }\end{array}$ & \begin{tabular}{|l} 
Preocuparse por \\
comunicar con \\
anticipación (cuando sea \\
posible) cualquier \\
procedimiento/ \\
intervención que se \\
realizará con el paciente, \\
y explicar su finalidad \\
\end{tabular} & 0,05 & $\begin{array}{c}p \\
=0,2925\end{array}$ & $\begin{array}{l}\text { No existe } \\
\text { diferencia } \\
\text { significativa }\end{array}$ \\
\hline $\begin{array}{l}\text { Enfermeros y } \\
\text { médicos } \\
\text { hablando } \\
\text { muy alto }\end{array}$ & $\begin{array}{l}\text { Estar atento de no hablar } \\
\text { muy alto en el área de } \\
\text { cuidados intensivos }\end{array}$ & 0,05 & $\begin{array}{c}p \\
=1,0000\end{array}$ & $\begin{array}{l}\text { No existe } \\
\text { diferencia } \\
\text { significativa }\end{array}$ \\
\hline $\begin{array}{l}\text { No tener } \\
\text { privacidad }\end{array}$ & $\begin{array}{l}\text { Preocuparse por la } \\
\text { privacidad al realizar una } \\
\text { intervención }\end{array}$ & 0,05 & $\begin{array}{c}\mathrm{p} \\
=0,2593\end{array}$ & $\begin{array}{c}\text { No existe } \\
\text { diferencia } \\
\text { significativa }\end{array}$ \\
\hline $\begin{array}{l}\text { Observar los } \\
\text { cuidados } \\
\text { médicos y de } \\
\text { enfermería } \\
\text { realizados } \\
\text { con otros } \\
\text { pacientes }\end{array}$ & $\begin{array}{l}\text { Estar siempre atento de } \\
\text { que un paciente no } \\
\text { observe los cuidados } \\
\text { médicos y de enfermería } \\
\text { realizados con otros } \\
\text { pacientes. }\end{array}$ & 0,05 & $\begin{array}{c}p \\
=0,3966\end{array}$ & $\begin{array}{l}\text { No existe } \\
\text { diferencia } \\
\text { significativa }\end{array}$ \\
\hline $\begin{array}{l}\text { No saber qué } \\
\text { día es hoy le } \\
\text { causa estrés }\end{array}$ & $\begin{array}{l}\text { Orientar al paciente } \\
\text { despierto respecto del día } \\
\text { de la semana y el horario. }\end{array}$ & 0,05 & $\begin{array}{c}p \\
=0,2020\end{array}$ & $\begin{array}{c}\text { No existe } \\
\text { diferencia } \\
\text { significativa }\end{array}$ \\
\hline Tener sed & $\begin{array}{l}\text { Preocuparse por la sed e } \\
\text { hidratación del paciente }\end{array}$ & 0,05 & $\begin{array}{c}p \\
=0,2001\end{array}$ & $\begin{array}{c}\text { No existe } \\
\text { diferencia } \\
\text { significativa }\end{array}$ \\
\hline Sentir dolor & $\begin{array}{l}\text { Tener algún método de } \\
\text { evaluación del dolor en la } \\
\text { institución que se } \\
\text { desempeña }\end{array}$ & 0,05 & $\begin{array}{c}p \\
=0,2280\end{array}$ & $\begin{array}{l}\text { No existe } \\
\text { diferencia } \\
\text { significativa }\end{array}$ \\
\hline
\end{tabular}

\section{DISCUSIÓN}

Mediante los datos obtenidos en el estudio, relacionados a las características sociodemográficas de los pacientes, se ha observado un promedio de edad de 50 años. Estudios anteriores encontraron relaciones importantes con la edad y nivel de estrés, siendo éstos inversamente proporcionales, es decir, cuanto más jóvenes son los pacientes, mayores son los niveles de estrés. ${ }^{(13)}$

No hay estudios que demuestren el índice más elevado de estrés en un sexo específico. En esta investigación, los pacientes fueron, en su mayoría, del sexo masculino, escogidos aleatoriamente, y teniendo en cuenta solo los aspectos de inclusión y exclusión. Aún así, otros estudios ${ }^{(6,9,10,13)}$ corroboran esta afirmación, presentando características similares de mayor índice de pacientes del sexo masculino internados en unidad de terapia intensiva.

Es importante resaltar el nivel de escolaridad elevado entre los participantes del estudio. El margen existente entre 9 y 12 años demuestra tener la enseñanza 
fundamental completa y la enseñanza media. Probablemente, esto haya disminuido los riesgos de incomprensión sobre los aspectos obtenidos con el cuestionario.

El estudio analizó los factores de estrés establecidos utilizando la EETI entre los pacientes participantes del estudio, aún así, el hecho de "Quedarse mirando al techo", sin poder realizar alguna actividad, relacionado, la mayoría de las veces, con la dificultad para pasar el tiempo, ha sido considerado como el principal estresor en el período de internación en la UTI.

Evidentemente los pacientes internados en la UTI enfrentan momentos de soledad, pues el tiempo autorizado para pasarlo en familia y con personas queridas es reducido. Para un paciente despierto, lúcido y orientado, muchas veces es difícil pasar el tiempo en vista de las diversas actividades que los mismos podrían estar aptos para realizar. Por esa razón, el día del paciente ingresado se hace largo, donde el silencio, la falta de actividad, la falta de alguien con quien conversar y la soledad, pueden deprimirlo. Tiene un largo tiempo para reflexionar respecto de su situación actual y los aspectos de su enfermedad. ${ }^{(14)}$

El segundo ítem evaluado como factor de estrés para el paciente fue: "No tener privacidad". Este hecho se contrapone a estudios anteriores ${ }^{(9,15)}$, en que la privacidad no fue considerada como un aspecto de preocupación del paciente ingresado. En esos casos, se relacionaron el hecho de que el paciente pasa por un momento de preocupación con los recursos de restauración de su salud y su estado actual, dejando de lado los pudores y las molestias al respecto. Sin embargo, en otro estudio $^{(16)}$, se ha constatado que la exposición de la intimidad y del cuerpo es una condición única para el paciente, pero una condición múltiple y común para los profesionales de la enfermería.

Se destaca la importancia de la ciencia del equipo multiprofesional, especializado en enfermería, por dedicar más tiempo al lado del paciente y en procedimientos de gran exposición y vulnerabilidad (ej.: baño), y preocuparse por las formas de disminuir la exposición, proporcionando al menos, un mínimo de privacidad.

Hubo un empate en los ítems indicados a continuación, aun considerados como factor de estrés destacados en "No saber qué día es hoy" y "Escuchar el gemido de otros pacientes". La cuestión de temporalidad para los pacientes es algo complejo, asociando el tiempo de ingreso con la iluminación artificial constante, la presencia de personas durante las 24 horas del día, el movimiento intenso, el horario de medicamentos durante toda la noche, la ausencia de ventanas/relojes, entre otros, causando así la pérdida referencial entre el día y la noche. Ese aspecto es importante, una vez que los pacientes pueden sentir que pierden el control de sus propias vidas cuando pierden sus referencias, teniendo la dificultad de situarse en el tiempo. ${ }^{(10,14,17)}$

Cabe recordar que es una importante responsabilidad de los profesionales que los asisten, poder situarlos en relación con el tiempo. Sucede que la mayoría de las veces, esos profesionales raramente lo perciben y se sensibilizan al respecto, pues conviven todas las mañanas en ese ambiente cerrado y están adaptados, sin considerar que este mismo ambiente es nuevo para el paciente, diferente, inédito y restrictivo. ${ }^{(1,17-19)}$

La estructura física de una UTI dificulta la privación de sonidos y ruidos. El paciente internado queda expuesto a sonidos provenientes de diversos medios, ya sean de 
pacientes o equipamientos. Tal vez la mayor molestia de los pacientes esté relacionada, no tanto con los decibeles alcanzados, sino con la proyección de sentimientos al pensar que otro ser humano esté viviendo un proceso de dolor e incomodidad, y tal vez trayéndolo a su realidad.

Contrariamente de otros estudios ${ }^{(9,20)}$, el "Sentir dolor" no fue una de las principales quejas de los pacientes. El dolor es una manifestación subjetiva, en la cual están involucrados, además de los factores físicos, los psíquicos y los culturales. ${ }^{(21)}$ Aún así, es generalmente una preocupación prioritaria para los profesionales de la salud.

Promover confort y alivio al dolor, no solo por cuestiones éticas y humanitarias, sino también debido a la comprobación de su interferencia en el estado físico, social y mental del paciente. ${ }^{(22)}$ Entonces, siempre se debe valorar y respetar la queja de dolor del paciente, y buscar medios de evaluación de la misma, debido a la disminución de la calidad de la evolución clínica del paciente, así como también la incomodidad que manifiesta. ${ }^{(21-23)}$

En el estudio se puede observar que la gran mayoría de los profesionales entrevistados se formó entre 6 y 10 años, en concordancia con el rango etario encontrado, pero se obtuvieron resultados significativos de tiempo de formación por encima de los 10 años. El rango de edad y el tiempo de formación son indicadores de experiencia de trabajo, esto se ve reflejado en la maduración, los conocimientos más evidentes y las capacidades en la vida diaria profesional. ${ }^{(23)}$

Vale resaltar de manera general, a los colaboradores del área de enfermería que realizan, de manera significativa, importantes acciones de humanización en su vida diaria laboral, evaluadas en las unidades de cuidados intensivos. Esto corrobora el hecho de que los pacientes estudiados hayan considerado a la UTI como un ambiente que va de no estresante a poco estresante.

Vale destacar las acciones a las que todos los colaboradores se refieren al realizarlas siempre, o en algunas ocasiones, como el de "Explicar siempre al paciente sobre el tratamiento/procedimiento realizado"; Preocuparse con "todo cuidado/ intervención y procedimiento con la privacidad del paciente" y "Estar siempre atento de que un paciente no observe los cuidados médicos y de enfermería realizados con otros pacientes".

Es importante considerar que la acción "menos" realizada por los colaboradores de enfermería que participaron del estudio es la de "Buscar orientar siempre al paciente despierto sobre qué día es hoy, qué horario, etc.". Esta cuestión de desorientación temporal es un factor establecido como estresor ${ }^{(24)}$ que el equipo de salud no debe descuidar.

Se ha observado que hubo un importante conflicto en la información referida a "Poseer algún método de evaluación de dolor en la institución en la que se desempeña”. Dentro de la misma institución, había divergencia en las respuestas. Conforme a la información manifestada por la institución, ambas poseían la Escala Visual Analógica (EVA), una escala unidimensional que evalúa las experiencias dolorosas.

En cuanto a la comparación de las respuestas de los pacientes con las de los colaboradores de enfermería, de acuerdo con las variables de los factores de estrés 
relacionados con el equipo, con el análisis estadístico de la Prueba Exacto de Fisher/Chi-Cuadrado, demostró algunas situaciones que presentaron diferencias significativas en la proporción de las respuestas, así como la variable en que "el enfermero no se presente por su nombre/ En todo primer contacto con el paciente, presentarse por su nombre; Enfermero y médico hablando muy alto/ Estar atento de no hablar muy alto en sala de cuidados críticos / Estar atento a los cuidados médicos $y$ de enfermería realizados en otros pacientes / Cuidar que un paciente no vea los cuidados médicos y de enfermería realizados en otros pacientes y Tener sed/ Preocuparse por preguntarle al paciente si tiene sed".

Respecto de los análisis de las variables de la Prueba de Mann-Whitney, del grado de estrés, que evaluó factores como No estresante, Poco estresante, Estresante y Muy estresante, no existió ninguna diferencia significativa en ninguna variable. Por lo tanto, se verifica que el profesional de enfermería tiene la capacidad de tener empatía, proyectándose y sensibilizándose a tal punto de saber cuándo y cuánto un factor es considerado estresante o no para el paciente.

\section{CONCLUSIONES}

Los factores que actúan como estresores en los pacientes internados en las dos UTI investigadas son principalmente quedarse mirando el techo, con dificultad para pasar el tiempo, no tener privacidad, no saber qué día es hoy y escuchar el gemido de otros pacientes.

Respecto de los mecanismos utilizados por el equipo de enfermería para amenizar los factores desencadenantes de estrés al paciente, se observó que, de manera general, los colaboradores de enfermería realizan de forma significativa, importantes acciones de humanización en su vida diaria laboral.

Luego de comparar las respuestas de los pacientes con las de los colaboradores de enfermería, se observó que el profesional de enfermería tiene la capacidad de proyectarse y sensibilizarse hasta el punto de saber cuándo y cuánto un determinado factor es estresante o no estresante en un paciente. Las necesidades de los pacientes ingresados son las mismas, en su mayoría, pero el grado de importancia para cada uno de ellos es variable. Se destaca la necesidad, cuando hablamos de humanización dentro del equipo, de un cuidado individual para cada paciente. Sobre todo, los profesionales están haciendo su trabajo de manera eficiente, pero este hecho no siempre es eficaz para sanar los problemas establecidos de todos los pacientes.

\section{REFERENCIAS}

1- Padilha KG, Vattimo MFF, Silva SC, Kimura M. Enfermagem em UTI: cuidando do paciente crítico. São Paulo: Manole; 2010.

2- Loures DL, Sant'Anna I, Baldotto CSR, Sousa EB, Nóbrega ACL. Estresse mental e sistema cardiovascular. Arq Bras Cardiol. 2002;78(5):525-30.

3-Greemberg JS. Administração do estresse. São Paulo: Manole, 2002.

4-Uhlig T, Kallus KW. Stress and stress disorders during and after intensive care. Cur Opin Anaesthesiol. 2004;17(1):131-5.

5- Rosa BA, Rodrigues RCM, Gallani MCBJ, Spana TM, Pereira CGS. Estressores em unidade de terapia intensiva: versão brasileira do The Environmental Stressor Questionnaire. Rev Esc Enferm USP. 2010;44(3):627-35. 
6- Bitencourt AGV, Neves FBCS, Dantas MP, Albuquerque LC, Melo RMV, Almeida AM, et al. Análise de estressores para o paciente em unidade de terapia intensiva. Rev Bras Ter Inten [online]. 2007;19(1):53-9.

7- BRASIL. Programa Nacional de Humanização da Assistência Hospitalar [Internet]. $\begin{array}{llllll}\text { Acesso } & \text { em } & 08 & \text { Set } 2010 . & \text { Disponível em: }\end{array}$ http://bvsms.saude.gov.br/bvs/publicacoes/pnhah01.pdf

8- Oriá MOB, Moraes LMP, Victor JF. A comunicação como instrumento do enfermeiro para o cuidado emocional do cliente hospitalizado [internet]. Rev Eletr Enferm.2004;6(2). Acesso em: 04 Out 2010. Disponível em: http://www.revistas.ufg.br/index.php/fen/article/viewArticle/808/921.

9-Marosti CA, Dantas RAS. Avaliação dos pacientes sobre os estressores em uma unidade coronariana. Acta Paul Enfermagem. 2006;19(2):190-5.

10- Haddad MCL, Alcantara C, Praes CS. Sentimentos e percepções do paciente no pós-operatório de cirurgia cardíaca, vivenciados em unidade de terapia intensiva. Cien Cuid e Saúde. 2005;4(1):65-73.

11- Souza WM, Silva F A, Teixeira D A, Machado R. C. Identificação dos principais estressores de pacientes internados em uma unidade coronariana. XIV encontro Latino Americano de Iniciação Científica. São José dos Campos, 2010.

12-Novaes MAFP, Aronovich A, Ferraz MB, Knobel E. Stressors in ICU: patients' evaluation. Intensive Care Med. 1997;23(1):1282-5.

13- Marosti CA, Dantas RAS. Relação entre estressores e características sociodemográficas e clínicas de pacientes internados em uma unidade coronariana. Rev Latino-am Enfermagem. 2006;14 (5).

14- Stumm EMF, Kuhn DT, Hildebrandt LM, Kirchner RM. Estressores vivenciados por pacientes em uma UTI. Cogitare Enf. 2008;13(4):499-506.

15-Salicio DMB, Gaiva MAM. Significado de humanização da assistência para enfermeiros que atuam em UTI. Ver Eletr Enfermagem. 2006;8(3):370-6. Acesso em 11 Maio 2011. Disponível em: http://www.fen.ufg.br/revista/revista8 3/v8n3a08.htm.

16- Baggio MA, Pomatti DM, Bettinelli LA, Erdmann AL. Privacidade em unidades de terapia intensiva: direitos dos pacientes e implicações para a enfermagem. Ver Bras Enfermagem. 2011;64(1):25-30.

17-Guirardello EB, Romero-Gabriel CAA, Pereira IC, Miranda AF. A percepção do paciente sobre sua permanência na unidade de terapia intensiva. Rev Esc Enferm USP. 1999; 33(2):123-9.

18- Vila VSC, Rossi LA. O significado cultural do cuidado humanizado em unidade de terapia intensiva: "muito falado e pouco vivido". Rev Latino-am Enfermagem. 2002;10(2):137-44.

19- Oliveira PS, Nóbrega MML, Silva ATMC, Filha MOF. Comunicação terapêutica em enfermagem revelada nos depoimentos de pacientes internados em centros de terapia intensiva [internet]. RevElet.Enfermagem. 2005;7(1):54-63. Acesso em: 08 Set 2010. Disponível em: http://www.fen.ufg.br/revista/revista7 1/original 05.htm

20- Gois CFL, Dantas RAS. Estressores em uma unidade pós-operatória de cirurgia torácica: avaliação da enfermagem. Rev Latino-am Enfermagem. 2004;12(1):22-7.

21- Pedroso RA, Celich KLS. Dor: quinto sinal vital, um desafio para o cuidar em enfermagem. Texto Contexto Enf. 2006;15(6):270-6.

22- Vila VSC, Mussi FC. O alívio da dor de pacientes no pós-operatório na perspectiva de enfermeiros de um centro de terapia intensiva. Rev Esc Enferm USP. 2001;35(3):300-7.

23. Brito MJR, Rocha AM, Ferreira VR. Análisis de los eventos adversos en una unidad de terapia intensiva neonatal como herramienta de gestión de calidad de la atención de enfermería. Enfermería Global [online]. 2009; 17: p. 0-0. 
24- Pinto JMS, Silva SF, Sampaio AP, Magalhães MS. A humanização da assistência na unidade de terapia intensiva na visão dos usuários. RPBS. 2008;21(2):121-7. 